\title{
NUMERI CARATTERISTICI DEI FLOCCULI D'IDROGENO E DI CALCIO E DEI FILAMENTI D'IDROGENO PER L'ANNO 1953
}

\author{
G. GoDoLI
}

Sono già state sottolineate in questa sede le ragioni che ci hanno spinto a riprendere in considerazione lopportunità di determinare l'attività dei fenomeni cromosferici [flocculi d'idrogeno, flocculi di calcio, filamenti d'idrogeno] mediante i numeri caratteristici $\left({ }^{1}\right)$.

Nella nota citata furono riportati i numeri caratteristici dei fenomeni cromosferici per gli anni 1950-51-52; qui, nelle seguenti tre tabelle di immediata interpretazione, sono riportati quelli per l'anno 1953.

I numeri caratteristici sono stati stimati sugli spettroeliogrammi eseguiti alla torre solare di Arcetri con l'immagine di $64 \mathrm{~mm}$; le caratteristiche strumentali e la tecnica delle osservazioni sono state descritte in altra sede $(-)$.

Sono state esaminate complessivamente 126 lastre in $H_{x}$ e 120 lastre in $K_{3.3}$.

La stima veniva eseguita con l'aiuto dei morlelli di Mt. Wilson. Per evidenti ragioni di uniformità è stata stimata prima tutta la serie dei numeri caratteristici per i flocculi d'idrogeno; quindi tutta la serie per i flocculi di calcio ed infine tutta la serie per i filamenti d'idrogeno. Lo scarto medio delle singole determinazioni is sempre stato inferiore a 0.2 unità.

Il contributo dei singoli osservatori nella raccolta delle lastre ̀̀ stato il seguente:

\begin{tabular}{|c|c|c|c|c|c|c|}
\hline Osserv & Fat ol & & & $H \propto$ & $\boldsymbol{K}_{2.3}$ & Totale \\
\hline Ahetti & . & $\cdot$ & . . & 4 & 3 & 7 \\
\hline Ballario. & $\cdot$ & $\cdot$ & . . & 88 & 86 & 174 \\
\hline Godoli . & $\cdot$ & . & . & 23 & 20 & 43 \\
\hline Righini. & $\cdot$ & . & . & t & 4 & 8 \\
\hline Rigutti . & . & . & . & 6 & 6 & 12 \\
\hline Romagnoli & . & . & . & 1 & 1 & 2 \\
\hline
\end{tabular}


Tabella I-a

Numeri caratteristici dei flocculi d'idrogeno per l'anno 1953.

\begin{tabular}{|c|c|c|c|c|c|c|c|c|c|c|c|c|}
\hline$\frac{\mathfrak{E}}{\stackrel{5}{5}}$ & & 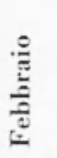 & $\stackrel{8}{\stackrel{E}{E}}$ & 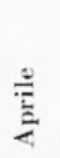 & 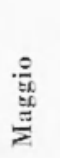 & 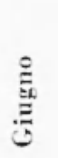 & $\stackrel{\varrho}{\stackrel{(v)}{\Xi}}$ & 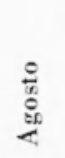 & 苛 & 䒕 & $\frac{\stackrel{0}{5}}{3}$ & 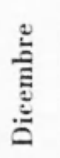 \\
\hline 1 & - & - & - & - & - & 0.7 & 0.1 & & 02 & & & \\
\hline 2 & - & 0.0 & - & - & 二 & - & 0.1 & 0.0 & 0.1 & 0.0 & - & - \\
\hline 3 & 0.1 & - & 0.0 & - & - & - & - & - & 0.0 & - & - & - \\
\hline 4 & - & 0.0 & 0.0 & - & - & - & - & - & 0.2 & - & - & 0.2 \\
\hline 5 & - & - & - & - & - & - & - & 0.2 & 0.1 & 8.0 & 0.3 & - \\
\hline 6 & - & - & 0.0 & - & - & - & - & 0.1 & - & 0.1 & - & - \\
\hline 7 & - & - & 0.0 & - & - & - & 0.0 & 0.3 & 0.3 & 0.2 & - & - \\
\hline 8 & - & - & - & 0.4 & - & - & 0.0 & 0.2 & 0.0 & 0.6 & - & - \\
\hline 9 & - & - & 0.0 & - & - & - & 0.9 & - & 0.1 & 0.4 & 0.0 & - \\
\hline 10 & 0.2 & - & 0.0 & - & - & - & - & 0.4 & - & - & 0.0 & - \\
\hline 11 & - & - & - & - & - & - & - & 0.5 & - & - & 0.0 & - \\
\hline 12 & - & - & 0.0 & - & - & - & - & 0.6 & 0.2 & - & 00 & - \\
\hline 13 & - & - & 0.0 & - & - & - & - & 1.2 & - & - & 0.0 & - \\
\hline 14 & - & - & - & - & - & - & 0.9 & 1.1 & - & - & - & - \\
\hline 15 & - & - & - & - & - & 0.0 & 0.0 & - & - & - & - & - \\
\hline 16 & 0.3 & - & $\overline{0 .}$ & $\overline{-}$ & 0.0 & - & 0.2 & - & - & - & - & 0.0 \\
\hline 17 & - & - & 0.0 & 0.0 & - & - & 0.4 & - & - & - & 0.0 & 0.0 \\
\hline 18 & $\overline{0}$ & - & 0.0 & 0.0 & - & - & 1.1 & - & - & - & 0.0 & - \\
\hline 19 & 0.1 & - & - & - & - & 0.3 & - & 0.3 & - & 0.1 & 0.0 & - \\
\hline 20 & 0.0 & - & 0.0 & 0.0 & - & - & 0.1 & 0.3 & - & - & 0.0 & - \\
\hline 21 & 0.0 & 0.0 & 0.0 & 0.0 & - & - & 0.0 & 0.3 & - & 0.9 & - & - \\
\hline 22 & 0.0 & - & - & - & - & 1.2 & 0.0 & - & - & - & - & - \\
\hline 23 & - & - & 0.0 & 0.3 & - & 1.1 & 0.0 & - & - & - & - & 0.0 \\
\hline 24 & - & - & - & - & - & - & 0.0 & 0.0 & - & - & - & - \\
\hline 25 & - & 0.0 & 0.1 & - & 0.3 & - & 0.0 & 00 & & - & - & - \\
\hline 26 & $\overline{-1}$ & 0.0 & 0.2 & - & 0.5 & 0.2 & - & - & - & - & 0.0 & - \\
\hline 27 & 0.0 & 0.0 & $\overline{-}$ & - & 0.8 & - & 0.0 & - & - & - & - & - \\
\hline 28 & - & 0.0 & 0.1 & 0.5 & $\overline{-}$ & - & 0.0 & ๑. 0 & - & - & - & - \\
\hline $\begin{array}{l}29 \\
20\end{array}$ & - & - & - & $\overline{0-7}$ & 0.6 & - & 0.0 & 0.0 & 0.2 & - & - & 0.1 \\
\hline $\begin{array}{l}70 \\
31\end{array}$ & E & $\bar{E}$ & E & $\begin{array}{l}0.7 \\
-\end{array}$ & $\overline{-}$ & - & $\begin{array}{l}0.0 \\
0.0\end{array}$ & $\overline{0.0}$ & - & $\overline{0.0}$ & 0.0 & $\overline{0.1}$ \\
\hline Medial & 0.1 & 0.0 & 0.0 & 0.2 & 0.4 & 0.6 & 0.1 & 0.3 & 0.1 & 0.2 & 0.0 & 0.1 \\
\hline \multicolumn{13}{|c|}{ Media annuale 0.16} \\
\hline
\end{tabular}




\begin{tabular}{|c|c|c|c|}
\hline \multirow{13}{*}{ 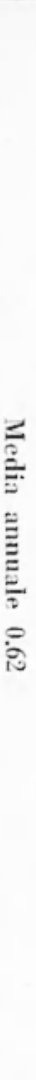 } & $\stackrel{\vec{D}}{\bar{\vdots}}$ & 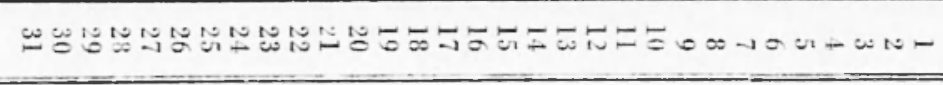 & Giorno \\
\hline & $\therefore$ & 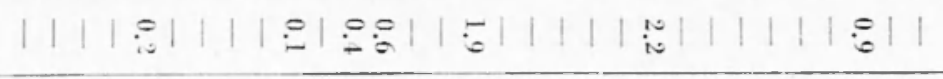 & Gennaio \\
\hline & is & 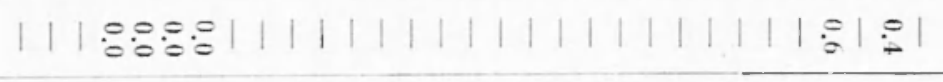 & Febbraio \\
\hline & $\stackrel{\check{\omega}}{i}$ & 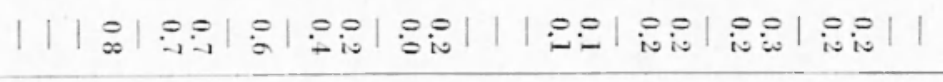 & Marzo \\
\hline & $\stackrel{\ominus}{-}$ & 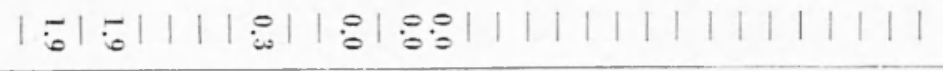 & Aprile \\
\hline & $\stackrel{\infty}{\infty}$ & ||$g \mid \overbrace{0}$ & Maggio \\
\hline & 5 & 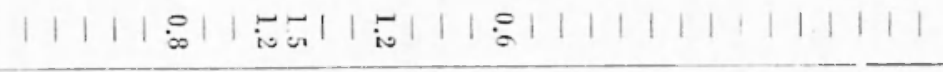 & Giugno \\
\hline & $\infty$ & 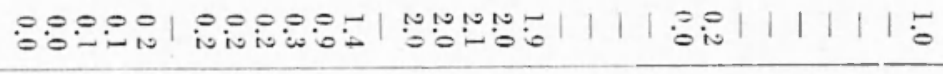 & Luglio \\
\hline & $\therefore$ & 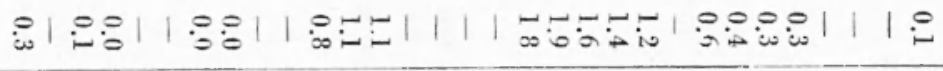 & Agosto \\
\hline & 5 & 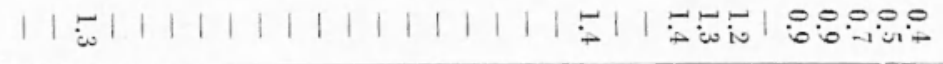 & Settembre \\
\hline & $\stackrel{\circ}{\infty}$ & 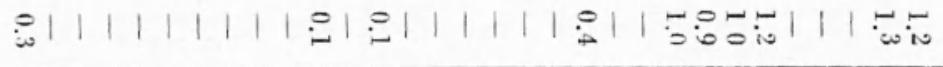 & Ottobre \\
\hline & is & 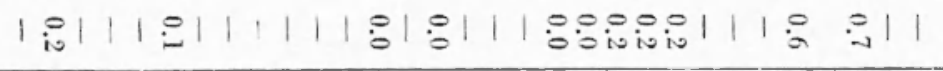 & Novembre \\
\hline & $\therefore$ & 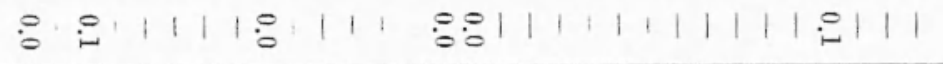 & Dicembre \\
\hline
\end{tabular}


'TABELla I-c

Numeri curatteristici dei filamenti d'idrogeno per lanno 1953.

\begin{tabular}{|c|c|c|c|c|c|c|c|c|c|c|c|c|}
\hline$\stackrel{\varrho}{\varrho}$ & 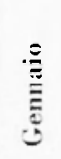 & & $\frac{O}{\tilde{E}}$ & $\stackrel{3}{\sum}$ & 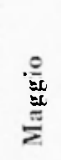 & 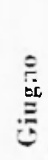 & 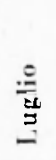 & $\begin{array}{l}\frac{e}{a} \\
\stackrel{\dot{c}}{\dot{c}} \\
<\end{array}$ & 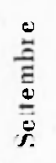 & 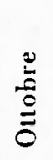 & 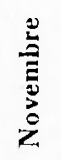 & 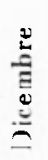 \\
\hline 1 & - & - & - & - & - & 0.9 & 0,3 & 0.1 & 110 & 0.2 & - & - \\
\hline 2 & - & 0.1 & - & - & - & - & - & - & 0.3 & 0.6 & - & - \\
\hline 3 & 0.1 & - & 0.2 & - & - & - & - & & 0.1 & - & - & - \\
\hline 4 & - & 0.8 & 0.1 & - & - & - & - & - & 0.3 & - & - & 0.4 \\
\hline 5 & - & - & - & - & - & - & - & 0.2 & 1.1 & 0.8 & 0.4 & - \\
\hline 6 & - & - & 0.2 & - & - & - & - & 0.0 & - & 1.2 & - & - \\
\hline 7 & - & - & 0.2 & - & - & - & 0.0 & 0.2 & 1.5 & 1.1 & - & - \\
\hline 8 & - & - & - & 0.8 & - & - & 0.0 & 0.1 & 0.0 & $1 . \cong$ & - & - \\
\hline 9 & - & - & 0.0 & - & - & - & 0.0 & - & $0 . ?$ & 1.8 & 0.3 & - \\
\hline 10 & 0.1 & - & 0.0 & - & - & - & - & 0.1 & - & - & 0.7 & - \\
\hline 11 & - & - & - & - & - & - & - & 0.3 & - & - & 0.6 & - \\
\hline 12 & - & - & 0.2 & - & - & - & - & 0.2 & 0.6 & - & 0.3 & - \\
\hline 13 & - & - & 0.0 & - & - & - & - & 0.4 & - & - & 0.0 & - \\
\hline 14 & - & - & - & - & - & - & 0.2 & 0.3 & - & - & - & - \\
\hline 1.5 & - & - & - & - & - & 0.2 & 0.2 & - & - & - & - & - \\
\hline 16 & 0.0 & - & - & - & 0.2 & - & 0.1 & - & - & - & - & 1.0 \\
\hline 17 & - & - & 0.0 & 0.0 & - & - & 0.3 & - & - & - & 0.1 & 1.4 \\
\hline 18 & - & - & 0.1 & 0.0 & - & - & 0.2 & - & - & - & 0.0 & \\
\hline 19 & 0.9 & - & - & - & - & 1.6 & - & 0.0 & - & 0.4 & 0.3 & - \\
\hline .0 & 0.4 & - & 0.0 & 0.2 & - & - & 0.1 & 0.1 & - & - & 0.2 & \\
\hline 21 & $0 . \prime$ & 0.0 & 0.0 & 0.2 & - & - & 0.1 & $\therefore .1$ & - & 0.0 & - & - \\
\hline 22 & 0.0 & - & - & - & - & 1.2 & 0.1 & - & - & - & - & \\
\hline 23 & - & - & 0.1 & 0.1 & - & 1.2 & 0,1 & - & - & - & - & 0.1 \\
\hline 2.4 & - & - & - & - & - & - & 0.4 & 0,0 & - & - & - & - \\
\hline 25 & - & 0.2 & 0.0 & - & 0.6 & - & 0.1 & 0.2 & - & - & - & - \\
\hline 26 & - & 0.2 & 0.0 & - & 1.3 & 0.9 & - & - & - & - & 0.6 & - \\
\hline 27 & 0.0 & 0.1 & - & - & 1.0 & - & 0.0 & - & - & - & - & - \\
\hline 23 & - & 0.1 & 0.0 & 0.2 & - & - & 0.9 & 0.0 & - & - & - & - \\
\hline 29 & - & - & - & - & 1.4 & - & 0.2 & 0.2 & 0.2 & - & - & 0.2 \\
\hline 30 & - & - & - & 0.4 & - & - & 0.0 & - & - & - & $0 . \overline{5}$ & - \\
\hline 31 & - & - & - & - & - & - & 0.1 & 0.4 & - & 1.2 & - & 0.3 \\
\hline Media & 0.2 & 0.2 & 0.1 & 0.2 & 0.9 & 1.0 & 0.2 & 0.2 & 0.5 & 0.8 & 0.3 & 0.6 \\
\hline \multicolumn{13}{|c|}{ Mcdia annuale 0.35} \\
\hline
\end{tabular}


Gli osservatori sono stati sempre coadiuvati dal sign. Romagnoli.

La distrihuzione mensile delle lastre ridotte $\dot{e}$ qui sotto indicata:

\begin{tabular}{|c|c|c|c|c|c|c|c|c|c|c|c|c|c|}
\hline & 1 & 2 & 3 & 4 & 5 & 6 & 7 & 8 & 9 & 10 & 11 & 12 \\
\hline $11 \alpha$ & 8 & 7 & 16 & 8 & 5 & 6 & 20 & 18 & 10 & 10 & 12 & 6 \\
\hline$K K_{*}$ & 7 & 6 & 16 & 6 & 6 & 5 & 19 & 18 & 10 & 10 & 11 & 6 \\
\hline
\end{tabular}

Firenze - Osservatorio astrofisico di Arcetri -- Centro di astrofisica del C.V.R. - Febbraio 1954.

\section{RI.ASSE:NTO}

Si danno i numeri caratteristici dei flocculi didrogeno " di calcio e dei filamenti d'idrogeno per l'anno 195.3, secondo le osservazioni eseguite alla torre solare di Arcetri.

\section{SUMMARY}

The character figures of Ca flocculi and of $\mathrm{H} \alpha$ bright and dark flocali are given according to the observations carried out at the solar tower of Arcetri.

\section{BIBI.IOC:RAFIA}

(1) G. Gobon, Numeri caratteristici dei locculi didrogeno e di calcio e dei filamenti didrogeno per gli anni 1950-51.52. Ann. Geof. 6, 199, (1953).

(-) G. Richini e G. Gobolı, Riduzione del materinle spettroeliografico raccolto alla torre solare di trcetri nel periodo 1932-1919. Men. SAI. 21. 333 (1950). 